\title{
Overregulation of microRNA-212 in the poor prognosis of esophageal cancer patients
}

\author{
B. Qi ${ }^{1}$, S.G. Liu ${ }^{1}$, X.G. Qin ${ }^{1}$, W.J. Yao ${ }^{1}$, J.G. Lu' ${ }^{1}$, L. Guo ${ }^{1}$, T.Y. Wang ${ }^{2}$, \\ H.C. $\mathrm{Li}^{1}$ and B.S. Zhao ${ }^{1}$ \\ ${ }^{1}$ Department of Thoracic Surgery, \\ First Affiliated Hospital of Xinxiang Medical University, \\ Weihui, China \\ ${ }^{2}$ Department of Biochemistry and Molecular Biology, \\ Xinxiang Medical University, Xinxiang, \\ Henan, China \\ Corresponding author: B.S. Zhao \\ E-mail: zhaobscn@126.com
}

Genet. Mol. Res. 13 (3): 7800-7807 (2014)

Received July 31, 2013

Accepted December 16, 2013

Published September 26, 2014

DOI http://dx.doi.org/10.4238/2014.September.26.18

\begin{abstract}
There have been few reports evaluating the expression and function of the microRNA miR-212 in esophageal cancer. The aim of this study was to investigate the relationship between miR-212 expression and clinicopathological factors and prognoses of esophageal cancer. MicroRNA was extracted from 46 esophageal cancer patients using the Taqman MicroRNA assay. All patients were at the same tumor node metastasis stage, but with different prognoses, and had all undergone surgery. The correlation between miR-212 expression and clinicopathological features was analyzed and the significance of miR-212 as a prognostic factor as well as its relationship with survival was determined. miR-212 expression was higher in patients with poor prognoses than in those with good prognoses $(\mathrm{P}<0.0001)$. Kaplan-Meier analysis results showed that the miR-212 expression level was significantly correlated with survival time $(\mathrm{P}=0.024)$. Patients with higher expression of miR-212 showed longer survival times. Cox multi-factor model analysis showed that miR-212
\end{abstract}


expression was significantly correlated with survival time $(\mathrm{P}=0.026)$. mir-212 is related with prognostic factors and survival time and may be a biomarker for esophageal cancer.

Key words: Esophageal cancer; Human microRNA-212; Prognostic factor

\section{INTRODUCTION}

Esophageal cancer (EC) is a type of malignant tumor that occurs in esophageal epithelial tissue and accounts for $2 \%$ of all malignant tumors. Approximately 200,000 people worldwide die from EC each year, and China is a particularly high incidence area. The high mortality rate from $\mathrm{EC}$ is second only to gastric cancer.

MicroRNAs (miRNAs), first discovered in 1993 (Lee et al., 1993), are small nonencoding RNAs 21-25 nucleotides in length. The human genome encodes more than 1000 miRNAs that downregulate target gene expression by interfering with mRNA translation (Berezikov et al., 2005). A previous study found that nearly half of all miRNA genes are located in fragile chromosomal sites or related genetic areas, regulate gene transcription, and function similarly to cancer genes and tumor-suppressor genes (Lanza et al., 2007). Numerous studies have established miRNAs as broad and powerful regulators of protein expression in physiology and disease (Liang et al., 2009a,b). Watson et al. (2007) found that miR-143 and miR-45 expression was significantly lower in colon cancer tissues, which was also observed in breast cancer and prostate cancer. Bloomston et al. (2007) analyzed 6 types of solid tumor (lung cancer, breast cancer, colon cancer, gastric cancer, prostate cancer, and pancreatic duct carcinoma) miRNA expression spectra using chromatin immunoprecipitation (ChIP) technology, and found that miR-21 expression was significantly high, suggesting that this miRNA has a wide range of action in carcinogenesis during the tumor formation process. Studies have shown that miR-21 downregulates the tumor suppressor gene tropomyosin 1 (TPM1). Schetter et al. (2008) found that miR-372 and miR-373 have oncogene effects in testicular tumor stem cells.

Some miRNAs directly participate in the initiation and development of tumors, and miRNA spectra are correlated with the diagnosis, staging, progression, and prognosis of EC (He et al., 2007; Zhou et al., 2009; Yang et al., 2013). Studies using microarray technology to examine the miRNA expression spectra of 31 EC tissue samples found that 46 miRNAs exhibited abnormal expression, 7 of which showed significant differences compared with those in normal tissues. Furthermore, the high expression of has-miR-103/107 is closely correlated with a low survival rate. To date, has-miR-335, has-miR-181d, has-miR-25, has-miR-7, and has-miR-495 have been confirmed to be correlated with histopathological stages of EC. In addition, abnormal miRNA expression spectra differ between esophageal squamous cell carcinoma (ESCC) tissues and esophageal adenocarcinoma (EAC) tissues (Lin et al., 2012; Xu et al., 2012).

In our hospital, EC patients at the same pathological stage that received the same surgical therapy by the same surgeon had distinct prognoses. The factors that led to the different postoperative prognoses were unknown. In our previous study, we found that miR-212 expression was related to the prognosis of ES patients who received the same therapy at same pathological stage. In this study, we examined the biological role of miR-212 expression in EC 
by investigating the relationship between miR-212 expression and clinicopathological characteristics as well as survival in EC patients.

\section{MATERIAL AND METHODS}

\section{Tissue samples}

We collected 46 clinical and pathological reports of patients at the same pathological stage but with different prognoses from stage 0 -III who received treatment at the First Affiliated Hospital of Xinxiang Medical University between September 2006 and December 2009. Among the 46 patients, 18 patients had good prognoses, and the other 28 cases had poor prognoses. In addition, paracancerous normal esophageal mucous membranes, $8 \mathrm{~cm}$ distant to the verge of the tumor tissue, were used as control samples. This study was conducted in accordance with the Declaration of Helsinki and with approval of the Ethics Committee of Xinxiang Medical University. Written informed consent was obtained from all participants.

\section{Sample treatment and RNA extraction}

Using a microtome, $10-\mu \mathrm{m}$ slices of formalin-fixed, paraffin-embedded tissue were placed in $1.5-\mathrm{mL}$ microcentrifuge tubes. Total RNA was extracted using TRI Reagent (Applied Biosystems; Foster City, CA, USA) according to manufacturer instructions. Briefly, 0.75 $\mathrm{mL}$ TRI Reagent was added and the sample was incubated at room temperature for $5 \mathrm{~min}$. After adding $0.5 \mathrm{~mL}$ isopropanol and mixing well, the samples were stored for $2 \mathrm{~h}$ at $-20^{\circ} \mathrm{C}$ and centrifuged at $13,800 \mathrm{~g}$ for $15 \mathrm{~min}$ at $4^{\circ} \mathrm{C}$. The supernatant was removed and $1 \mathrm{~mL} 70 \%$ ice-cold ethanol was added to dissolve the pellet. The sample was re-centrifuged and the supernatant was discarded again. The final pellet was dissolved in $50 \mu \mathrm{L}$ diethylpyrocarbonatedistilled water and RNA yield was determined using an ultraviolet (UV) spectrophotometer.

\section{Real-time quantitative reverse transcription-polymerase chain reaction (RT-q CR)}

DNA amounts of $0.5-1 \mu \mathrm{g}$ were used in the reverse transcriptase reaction. After reverse transcription, a TaqMan MicroRNA Assay (Applied Biosystems) was performed according to the manufacturer protocol to detect miR-212 expression using miR-197 as a primer the small nuclear RNA RNU6 (Assay ID: 001093) was used as a control. The relative quantitative method was used. Gene expression was quantified based on the following formula: $F=2^{-\Delta \Delta C t}$, where $\Delta \Delta \mathrm{Ct}=$ [mean cycle threshold $(\mathrm{Ct})$ of the target gene in the test sample - mean $\mathrm{Ct}$ of the housekeeping gene in the test sample] - (mean Ct of the target gene in the control sample - that of the housekeeping gene in the control sample). A higher $\mathrm{F}$ value indicated higher relative expression.

\section{Statistical analysis}

The relationship between the miR-212 expression level and clinicopathological features of the patients was analyzed using the chi-square test. We used SPSS 17.0 to establish the database and analyze the data (SPSS, Inc.; Chicago, IL, USA). Kaplan-Meier analysis was used to analyze the relationship between microRNA expression and survival time. A Cox 
regression model was used to analyze the influence of the related factors on the survival time of patients with EC. $\mathrm{P}<0.05$ was considered to be statistically significant.

\section{RESULTS}

\section{Patient characteristics}

Forty-six patients with ESCC without preoperative radiotherapy and chemotherapy were enrolled in this study. All patients underwent surgical resection at the Department of Thoracic Surgery, the First Affiliated Hospital of Xinxiang Medical University. All postoperative samples were confirmed for ESCC by histopathological examination. Tumors were staged according to the International Union against Cancer (UICC) staging criteria for EC, (Sobin et al., 2002). The study patients included 23 males and 23 females aged 45-71 years (median, 59 years). Nineteen tumors were $>5 \mathrm{~cm}$ and 27 cases were $\leq 5 \mathrm{~cm}$. Eleven cases of tumor differentiation were well differentiated, 19 cases showed moderate differentiation, and 16 cases were poorly differentiated. Nineteen cases showed lymph node metastasis, and 27 cases were not in metastasis. One case was in stage $0 ; 6$ cases were in stage I; 20 cases were in stage IIa; 1 case was in stage IIb; and 18 cases were in stage III. There were 28 early recurrence patients (tumor recurrence within 1 year after operation) and 18 non-recurrence patients (patients who survived more than 5 years without recurrence). The patients' characteristics are outlined in Table 1.

\begin{tabular}{lc} 
Table 1. Patient characteristics. & \\
\hline Character & No. of patients (\%) \\
\hline All patients & $46(100.0)$ \\
Gender & $23(50.0)$ \\
Male & $23(50.0)$ \\
Female & $21(45.7)$ \\
Age & $25(54.3)$ \\
$\geq 60$ years & $4(8.7)$ \\
$<60$ years & $35(76.1)$ \\
Site of tumor & $7(15.2)$ \\
Upper thoracic & $27(58.7)$ \\
Middle thoracic & $19(41.3)$ \\
Lower thoracic & $11(16.3)$ \\
Size of tumor & $19(41.3)$ \\
$\leq 5$ cm & $16(34.8)$ \\
$>5$ cm & \\
Differentiation & $27(58.7)$ \\
Well & $19(41.3)$ \\
Moderate & \\
Poor & $8(17.4)$ \\
Lymph node metastasis & $6(13.0)$ \\
Negative & $32(69.6)$ \\
Positive & \\
Depth of invasion & \\
Tis, T1 & $7(15.2)$ \\
T2 & $21(45.7)$ \\
T3 & $18(39.1)$ \\
Stage (TNM) & \\
III & $28(60.9)$ \\
IIII & $18(39.1)$ \\
Recurrence & \\
Early recurrence & \\
Non recurrence & \\
\hline
\end{tabular}




\section{miR-197 expression in EC}

The results showed that the miR-212 expression level decreased by 2.20 -fold in the samples with good prognoses compared with the samples with poor prognoses. The mean expression levels of the samples with good and poor prognoses were 5.076 \pm 5.744 and $11.139 \pm$ 9.115, respectively, and the difference was statistically significant (Figure 1, P $<0.05$ ).

A
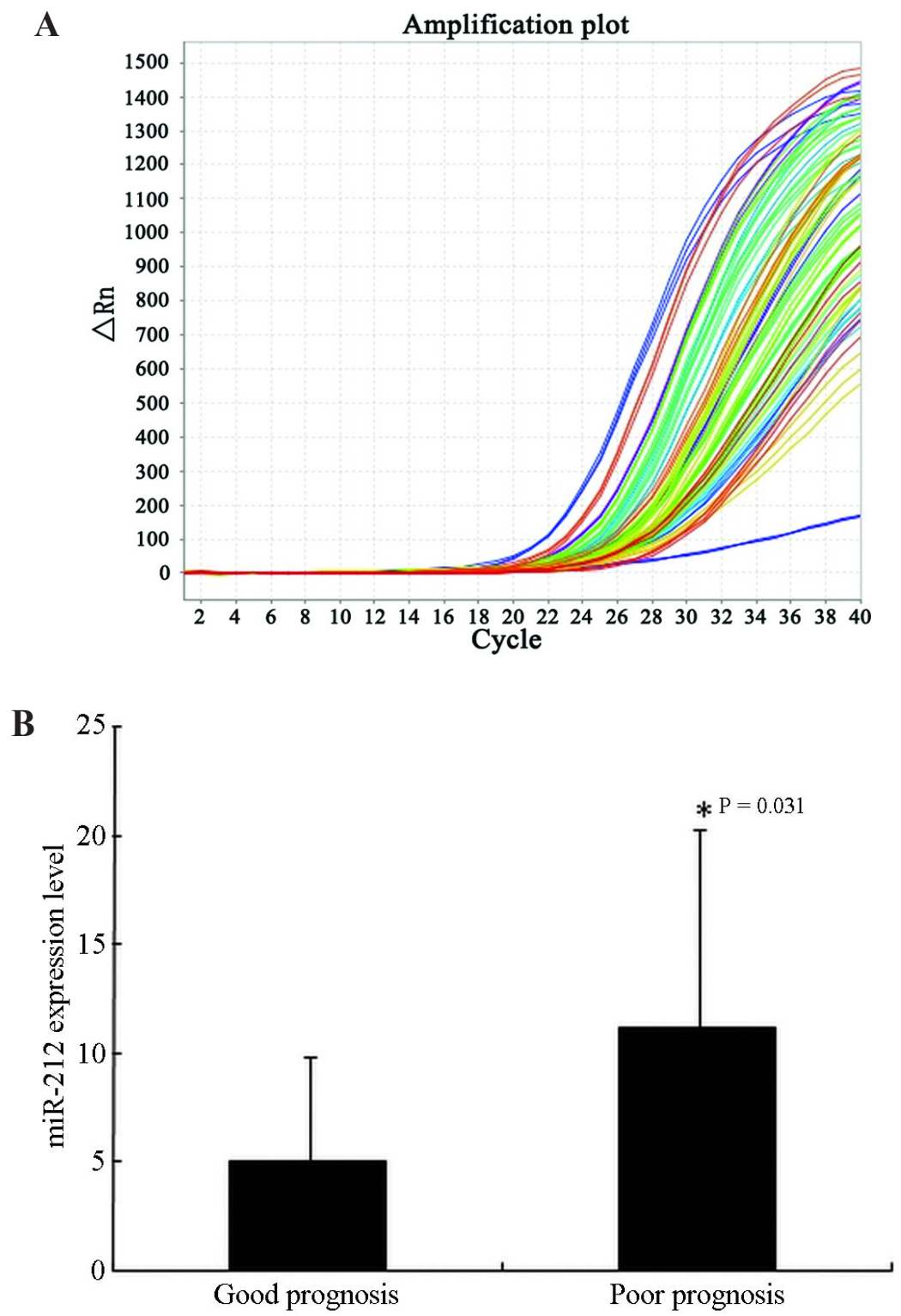

Figure 1. A. Amplification plot with $\Delta$ ct values of miR-212 in esophageal cancer with good and poor prognosis. B. Comparison of miR-212 expression in esophageal cancer between the sample with good and poor prognosis. 


\section{Association of miR-212 expression with prognosis and survival of EC patients}

The Kaplan-Meier analysis results showed that the miR-212 expression level was significantly correlated with survival time $(\mathrm{P}=0.024)$; patients with higher miR-212 expression had longer survival times. Cox single-factor related risk analysis results showed that patient prognosis $(\mathrm{P}=0.000)$, TNM stage $(\mathrm{P}=0.023)$, invasion depth $(\mathrm{P}=0.010)$, tumor size $(\mathrm{P}=0.006)$, miR-212 expression $(\mathrm{P}=0.007)$, and patient survival time were significantly correlated. Cox multi-factor model analysis showed that patient prognosis $(\mathrm{P}=0.002)$, tumor size $(\mathrm{P}=0.001)$, miR-212 expression $(\mathrm{P}=0.026)$, and patient survival time were significantly correlated, with corresponding risks of 7.405, 1.399, and 2.785 (Figure 2).

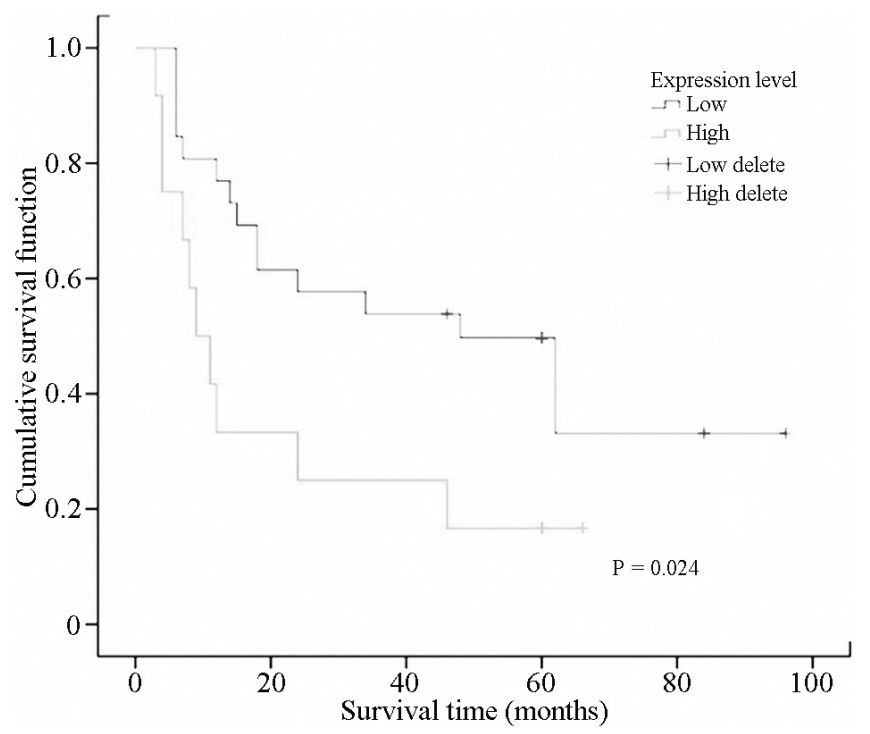

Figure 2. Relationship between miR-212 expression and survival time.

\section{DISCUSSION}

The number of studies examining the relationship between miRNAs and cancers has increased recently, and the potential of using miRNAs as novel biomarkers is growing (Mathé et al., 2009; Yu et al., 2010). However, there have been no reports on miR-212 in EC. In the present study, we found that miR-212 expression differed in patients of the same pathological stage but with different prognoses that had received the same treatment. MiR-212 expression was lower in samples with good prognoses and higher in samples with poorer prognoses. Moreover, statistical analysis showed that miR-212 expression was significantly correlated with survival time.

Consistent with our results, previous studies found that miR-212 expression is decreased in cancer, including gastric cancer (Wada et al., 2010; Wu et al., 2011; Xu et al., 2011), lung cancer (Incoronato et al., 2011), and myelogenous leukemia cell (Incoronato et al., 2011). This is explained by the hypermethylation of $\mathrm{CpG}$ islands (CPI) in the promoter regions of miR-212, which leads to lower expression of miR-212 in gastric cell strains. MiR-212 expres- 
sion was significantly recovered after treatment with the methylation inhibitor 5-Aza-dC (Xu et al., 2011). Downregulation of miR-212 may be related to gastric carcinogenesis through its target genes, such as MECP2 (Wada et al., 2010). However, Incoronato et al. (2011) found that histone modifications rather than DNA hypermethylation as epigenetic events regulate miR212 levels in non-small cell lung carcinoma (NSCLC). MiR-212 silencing in vivo is closely associated with the severity of NSCLC.

Previous studies demonstrated that miR-212 expression was significantly elevated compared with controls. Scapoli et al. (2010) found that miR-212 was significantly overexpressed in oral squamous cell carcinoma using microarray analysis. Park et al. (2011) reported that 2 miRNAs located on chromosome 17p13, miR-132 and miR-212, are overexpressed in pancreatic adenocarcinoma tissues. Therefore, miR-212 may have tumor-promoting properties. The tumor suppressor PTCH1 is a functional target of miR-212 and may be responsible for the effect of miR-212 on cell proliferation.

miR-212 was demonstrated to be involved in tumorigenesis, and the oncogenic activity of miR-212 in NSCLC cells was due, in part, to the suppression of PTCH1 (Li et al., 2012). However, there have been no reports examining the relationship between miR-212 and EC.

There were several limitations to our study. Although patient selection was consecutive from 2006 to 2009, lack of materials resulted in a relatively small population for analysis, and therefore selection bias may have occurred. Because of the small population and short follow-up period, the number of deaths was low, which may have influenced our analysis. In addition, there is currently no international standardized definition for methods of analysis and determining expression levels for miRNAs. Some studies used the mean relative expression value (RQ) to define high and low expression, while other studies used different definitions to group expression levels. This discordant analysis can lead to disagreement among studies.

In conclusion, our study showed that miR-212 is overexpressed in EC patients with poor prognoses but at the same pathological stage and having received the same surgical therapy as patients with good prognoses. High miR-212 expression was associated with poor prognostic factors and poor survival. These findings suggest that miR-212 has profound potential as a marker for predicting the outcome, treatment response, and treatment method for EC.

\section{REFERENCES}

Berezikov E, Guryev V, van de Belt J, Wienholds E, et al. (2005). Phylogenetic shadowing and computational identification of human microRNA genes. Cell 120: 21-24.

Bloomston M, Frankel WL, Petrocca F, Volinia S, et al. (2007). MicroRNA expression patterns to differentiate pancreatic adenocarcinoma from normal pancreas and chronic pancreatitis. JAMA 297: 1901-1908.

He B, Pan Y, Cho WC, Xu Y, et al. (2007). The association between four genetic variants in microRNAs (rs11614913, rs2910164, rs3746444, rs2292832) and cancer risk: evidence from published studies. PLoS One 7: e49032.

Incoronato M, Urso L, Portela A, Laukkanen MO, et al. (2011). Epigenetic regulation of miR-212 expression in lung cancer. PLoS One 6: e27722.

Lanza G, Ferracin M, Gafa R, Veronese A, et al. (2007). mRNA/microRNA gene expression profile in microsatellite unstable colorectal cancer. Mol. Cancer 6: 54.

Lee RC, Feinbaum RL and Ambros V (1993). The C. elegans heterochronic gene lin-4 encodes small RNAs with antisense complementarity to lin-14. Cell 75: 843-854.

Li Y, Zhang D, Chen C, Ruan Z, et al. (2012). MicroRNA-212 displays tumor-promoting properties in non-small cell lung cancer cells and targets the hedgehog pathway receptor PTCH1. Mol. Biol. Cell 23: 1423-1434.

Liang M (2009a). MicroRNA: a new entrance to the broad paradigm of systems molecular medicine. Physiol. Genomics 38: 113-115. 
Liang M, Liu Y, Mladinov D, Cowley AW Jr, et al. (2009b). MicroRNA: a new frontier in kidney and blood pressure research. Am. J. Physiol. Renal Physiol. 297: F553-F558.

Lin RJ, Xiao DW, Liao LD, Chen T, et al. (2012). MiR-142-3p as a potential prognostic biomarker for esophageal squamous cell carcinoma. J. Surg. Oncol. 105: 175-182.

Mathé EA, Nguyen GH, Bowman ED, Zhao Y, et al. (2009). MicroRNA expression in squamous cell carcinoma and adenocarcinoma of the esophagus: associations with survival. Clin. Cancer Res. 15: 6192-6200.

Park JK, Henry JC, Jiang J, Esau C, et al. (2011). miR-132 and miR-212 are increased in pancreatic cancer and target the retinoblastoma tumor suppressor. Biochem. Biophys. Res. Commun. 406: 518-523.

Scapoli L, Palmieri A, Lo ML, Pezzetti F, et al. (2010). MicroRNA expression profiling of oral carcinoma identifies new markers of tumor progression. Int. J. Immunopathol. Pharmacol. 23: 1229-1234.

Schetter AJ, Leung SY, Sohn JJ, Zanetti KA, et al. (2008). MicroRNA expression profiles associated with prognosis and therapeutic outcome in colon adenocarcinoma. JAMA 299: 425-436.

Sobin LH, Gospodarowicz MK and Wittekind C (2002). TNM Classification of Malignant Tumours (UICC International Union Against Cancer). Wiley-Blackwell Publish House, New York.

Wada R, Akiyama Y, Hashimoto Y, Fukamachi H, et al. (2010). miR-212 is downregulated and suppresses methyl-CpGbinding protein MeCP2 in human gastric cancer. Int. J. Cancer 127: 1106-1114.

Watson DI, Wijnhoven BPL, Michael MZ, Mayne GC, et al. (2007). MicroRNA expression profiles in Barrett's oesophagus. ANZ J. Surg. 77: 1-4

Wu WY, Xue XY, Chen ZJ, Han SL, et al. (2011). Potentially predictive microRNAs of gastric cancer with metastasis to lymph node. World J. Gastroenterol. 17: 3645-3651.

Xu L, Wang F, Xu XF, Mo WH, et al. (2011). Downregulation of miR-212 expression by DNA hypermethylation in human gastric cancer cells. Med. Oncol. 28 (Suppl 1): S189-S196.

Xu X, Chen Z, Zhao X, Wang J, et al. (2012). MicroRNA-25 promotes cell migration and invasion in esophageal squamous cell carcinoma. Biochem. Biophys. Res. Commun. 421: 640-645.

Yang M, Liu R, Sheng J, Liao J, et al. (2013). Differential expression profiles of microRNAs as potential biomarkers for the early diagnosis of esophageal squamous cell carcinoma. Oncol. Rep. 29: 169-176.

Yu Z, Baserga R, Chen L, Wang C, et al. (2010). microRNA, cell cycle, and human breast cancer. Am. J. Pathol. 176: 1058-1064.

Zhou ZQ, Cao WH, Xie JJ, Lin J, et al. (2009). Expression and prognostic significance of THBS1, Cyr61 and CTGF in esophageal squamous cell carcinoma. BMC Cancer 9: 291. 